PROCEEDINGS OF THE

AMERICAN MATHEMATICAL SOCIETY

Volume 129, Number 3, Pages 815-821

$\mathrm{S} 0002-9939(00) 05621-5$

Article electronically published on September 19, 2000

\title{
A NUMERICAL RANGE CHARACTERIZATION OF UNIFORMLY SMOOTH BANACH SPACES
}

\author{
ANGEL RODRIGUEZ PALACIOS
}

(Communicated by Dale Alspach)

\begin{abstract}
We prove that a Banach space $X$ is uniformly smooth if and only if, for every $X$-valued bounded function $f$ on the unit sphere of $X$, the intrinsic numerical range of $f$ is equal to the closed convex hull of the spatial numerical range of $f$.
\end{abstract}

\section{INTRODUCTION}

Let $X$ be a Banach space over $\mathbb{K}(=\mathbb{R}$ or $\mathbb{C})$. We denote by $S_{X}, B_{X}$, and $X^{*}$ the unit sphere of $X$, the closed unit ball of $X$, and the dual space of $X$, respectively. For $u$ in $S_{X}$, we denote by $D(X, u)$ the set of all states of $X$ relative to $u$, namely

$$
D(X, u):=\left\{\phi \in S_{X^{*}}: \phi(u)=1\right\},
$$

and then, for $x$ in $X$, we define the numerical range $V(X, u, x)$ of $x$ relative to $u$ as the nonempty, convex, and compact subset of $\mathbb{K}$ given by the equality

$$
V(X, u, x):=\{\phi(x): \phi \in D(X, u)\} .
$$

Given a mapping $f$ from $S_{X}$ into $X$, we can consider the so-called spatial $n u$ merical range $W(f)$ of $f$, namely

$$
W(f):=\bigcup\left\{V(X, u, f(u)): u \in S_{X}\right\} .
$$

If the mapping $f$ above is bounded, then it also has an intrinsic numerical range $V(f)$, given by the equality

$$
V(f):=V\left(B\left(S_{X}, X\right), \mathbf{1}, f\right)
$$

where $B\left(S_{X}, X\right)$ denotes the Banach space of all bounded functions from $S_{X}$ to $X$, and 1 stands for the natural embedding $S_{X} \hookrightarrow X$. In this case we have the inclusion

$$
\overline{\mathrm{co}} W(f) \subseteq V(f),
$$

where $\overline{\text { co }}$ means closed convex hull. (Indeed, for $u$ in $S_{X}$ and $\phi$ in $D(X, u)$, the mapping $g \rightarrow \phi(g(u))$ from $B\left(S_{X}, X\right)$ to $\mathbb{K}$ is an element of $D\left(B\left(S_{X}, X\right), \mathbf{1}\right)$.) The inclusion above is known to be an equality whenever either $f$ is (the restriction to $S_{X}$ of) a continuous linear operator on $X[\underline{6}$, or $\mathbb{K}=\mathbb{C}$ and $f$ is (the restriction to $S_{X}$ of) a uniformly continuous function on $B_{X}$ which is holomorphic on the interior of $B_{X}[5]$.

Received by the editors October 19, 1998 and, in revised form, May 24, 1999.

2000 Mathematics Subject Classification. Primary 46B04, 46B20.

(C)2000 American Mathematical Society 
The aim of this note is to show that the equality $\overline{\mathrm{co}} W(f)=V(f)$ can hold for every $f$ in $B\left(S_{X}, X\right)$, but only if $X$ lies in a very restrictive class of Banach spaces. Actually, we prove that the equality $\overline{\mathrm{co}} W(f)=V(f)$ holds for every $f$ in $B\left(S_{X}, X\right)$ if and only if $X$ is uniformly smooth. Note that every uniformly smooth Banach space is super-reflexive, and that, in fact, super-reflexivity is the isomorphic side of the uniform smoothness, i.e., every super-reflexive Banach space has a uniformly smooth equivalent renorming (see for instance [2]).

\section{The REsults}

Let $X$ be a Banach space over $\mathbb{K}(=\mathbb{R}$ or $\mathbb{C})$, and $u$ an element in $S_{X}$. For $x$ in $X$, the function $\alpha \rightarrow\|u+\alpha x\|$ from $\mathbb{R}$ to $\mathbb{R}$ is convex, hence there exists the number

$$
M^{u}(x):=\lim _{\alpha \rightarrow 0^{+}} \frac{\|u+\alpha x\|-1}{\alpha}=\inf \left\{\frac{\|u+\alpha x\|-1}{\alpha}: \alpha \in \mathbb{R}^{+}\right\},
$$

and it is known that the equality

$$
M^{u}(x)=\max \{\operatorname{Re}(\lambda): \lambda \in V(X, u, x)\}
$$

holds (see for instance [3, Theorem V.9.5]). For $\alpha$ in $\mathbb{R}^{+}$, we put

$$
\varphi(X, u, \alpha):=\sup \left\{\frac{\|u+\alpha x\|-1}{\alpha}-M^{u}(x): x \in B_{X}\right\} .
$$

Theorem 1. Let I be an infinite set, $\left\{X_{i}\right\}_{i \in I}$ a family of Banach spaces over $\mathbb{K}$, and, for each $i$ in $I$, let $u_{i}$ be a fixed norm-one element in $X_{i}$. Denote by $Y$ the $l_{\infty}$-sum of the family $\left\{X_{i}\right\}_{i \in I}$, and by $u$ the element of $S_{Y}$ given by $u(i):=u_{i}$ for every $i$ in $I$. Then the following assertions are equivalent:

i) the equality

$$
V(Y, u, y)=\overline{\mathrm{co}}\left(\bigcup\left\{V\left(X_{i}, u(i), y(i)\right): i \in I\right\}\right)
$$

holds for every $y$ in $Y$.

ii) $\lim _{(i, \alpha) \rightarrow(\infty, 0)} \varphi\left(X_{i}, u(i), \alpha\right)=0$.

In assertion ii) above, the symbol $\lim _{(i, \alpha) \rightarrow(\infty, 0)}$ means the limit along the filter basis on $I \times \mathbb{R}^{+}$consisting of all subsets of $I \times \mathbb{R}^{+}$of the form $J \times(0, \delta)$, where $J$ is a co-finite subset of $I$ and $\delta$ is a positive number. We also note that, if the set $I$ in the theorem fails to be infinite, then, for every $y$ in $Y$, the equality

$$
V(Y, u, y)=\operatorname{co}\left(\bigcup\left\{V\left(X_{i}, u(i), y(i)\right): i \in I\right\}\right)
$$

is automatically true.

Proof of Theorem 1. ii) $\Rightarrow$ i) Fix $y$ in $S_{Y}$, and let $\varepsilon$ be a positive number. By assumption ii), there exists $\delta>0$, and a co-finite subset $J$ of $I$, such that the inequality

$$
\frac{\left\|u(j)+\alpha x_{j}\right\|-1}{\alpha}-M^{u(j)}\left(x_{j}\right)<\varepsilon
$$

holds whenever $j$ is in $J, x_{j}$ is in $B_{X_{j}}$ for such a $j$, and $0<\alpha<\delta$. For $k$ in $I \backslash J$, we can choose $\delta_{k}>0$ such that

$$
\frac{\|u(k)+\alpha y(k)\|-1}{\alpha}-M^{u(k)}(y(k))<\varepsilon
$$


whenever $0<\alpha<\delta_{k}$. By putting $\rho:=\min \left\{\delta, \min \left\{\delta_{k}: k \in I \backslash J\right\}\right\}$, it follows that the inequality

$$
\frac{\|u(i)+\alpha y(i)\|-1}{\alpha}<M^{u(i)}(y(i))+\varepsilon
$$

is true for every $i$ in $I$ and $0<\alpha<\rho$. Therefore, for $0<\alpha<\rho$ we have

$$
\frac{\|u+\alpha y\|-1}{\alpha} \leq \sup \left\{M^{u(i)}(y(i)): i \in I\right\}+\varepsilon,
$$

and, by letting $\alpha \rightarrow 0$, we obtain

$$
M^{u}(y) \leq \sup \left\{M^{u(i)}(y(i)): i \in I\right\}+\varepsilon .
$$

In view of the arbitrariness of $\varepsilon$, we actually have

$$
M^{u}(y) \leq \sup \left\{M^{u(i)}(y(i)): i \in I\right\},
$$

so that, replacing $y$ by $z y$ with $z$ in $S_{\mathbb{K}}$, the inclusion

$$
V(Y, u, y) \subseteq \overline{\mathrm{co}}\left(\bigcup\left\{V\left(X_{i}, u(i), y(i)\right): i \in I\right\}\right)
$$

follows. The reverse inclusion is trivial.

i) $\Rightarrow$ ii) Assume that ii) is not true. Then there is $\varepsilon>0$ such that, for every co-finite subset $J$ of $I$, and for every $\delta>0$, there exists $0<\alpha<\delta, j$ in $J$, and $x_{j}$ in $B_{X_{j}}$ such that

$$
\frac{\left\|u(j)+\alpha x_{j}\right\|-1}{\alpha}-M^{u(j)}\left(x_{j}\right) \geq \varepsilon .
$$

Take $0<\alpha_{1}<1, i(1)$ in $I$, and $x_{i(1)}$ in $B_{X_{i(1)}}$ such that

$$
\frac{\left\|u(i(1))+\alpha_{1} x_{i(1)}\right\|-1}{\alpha_{1}}-M^{u(i(1))}\left(x_{i(1)}\right) \geq \varepsilon .
$$

Assume that, for some $n$ in $\mathbb{N}, \alpha_{1}, \ldots, \alpha_{n}, i(1), \ldots, i(n), x_{i(1)}, \ldots, x_{i(n)}$ have been found such that $0<\alpha_{m}<\frac{1}{m}, i(m)$ is in $I, x_{i(m)}$ belongs to $X_{i(m)}$,

$$
\frac{\left\|u(i(m))+\alpha_{m} x_{i(m)}\right\|-1}{\alpha_{m}}-M^{u(i(m))}\left(x_{i(m)}\right) \geq \varepsilon
$$

(for all $m$ in $\{1, \ldots, n\}$ ), and $i(m) \neq i\left(m^{\prime}\right)$ (for all $m, m^{\prime}$ in $\{1, \ldots, n\}$ with $m \neq m^{\prime}$ ). Then we can choose $0<\alpha_{n+1}<\frac{1}{n+1}, i(n+1)$ in $I \backslash\{i(1), \ldots, i(n)\}$, and $x_{i(n+1)}$ in $B_{X_{i(n+1)}}$ satisfying

$$
\frac{\left\|u(i(n+1))+\alpha_{n+1} x_{i(n+1)}\right\|-1}{\alpha_{n+1}}-M^{u(i(n+1))}\left(x_{i(n+1)}\right) \geq \varepsilon .
$$

In this way, we have constructed a sequence $\left\{\left(\alpha_{n}, i(n), x_{i(n)}\right)\right\}_{n \in \mathbb{N}}$ with $\alpha_{n} \in \mathbb{R}^{+}$, $\left\{\alpha_{n}\right\} \rightarrow 0, i(n) \in I, i(n) \neq i(m)$ for $n \neq m, x_{i(n)} \in B_{X_{i(n)}}$, and

$$
\frac{\left\|u(i(n))+\alpha_{n} x_{i(n)}\right\|-1}{\alpha_{n}}-M^{u(i(n))}\left(x_{i(n)}\right) \geq \varepsilon .
$$

Put $r:=\limsup _{n \rightarrow \infty}\left\{M^{u(i(n))}\left(x_{i(n)}\right)\right\}$, so that, by discarding a finite number of terms in the sequence $\left\{\left(\alpha_{n}, i(n), x_{i(n)}\right)\right\}$, we can assume that, in addition to the above properties, we have

$$
M^{u(i(n))}\left(x_{i(n)}\right) \leq r+\frac{\varepsilon}{2}
$$


for every $n$. Now, consider the element $y$ in $Y$ defined by $y(i)=x_{i(n)}$ if $i=i(n)$ for some $n$, and $y(i)=r u(i)$ otherwise. Then, for every $n$ in $\mathbb{N}$ we have

$$
\begin{aligned}
\frac{\left\|u+\alpha_{n} y\right\|-1}{\alpha_{n}} & \geq \frac{\left\|u(i(n))+\alpha_{n} y(i(n))\right\|-1}{\alpha_{n}} \\
& =\frac{\left\|u(i(n))+\alpha_{n} x_{i(n)}\right\|-1}{\alpha_{n}} \geq M^{u(i(n))}\left(x_{i(n)}\right)+\varepsilon .
\end{aligned}
$$

By taking upper limits as $n \rightarrow \infty$, we obtain

$$
M^{u}(y) \geq r+\varepsilon .
$$

On the other hand, the definition of $y$ yields the inequality

$$
\sup \left\{M^{u(i)}(y(i)): i \in I\right\} \leq r+\frac{\varepsilon}{2} \text {. }
$$

It follows that

$$
\sup \left\{M^{u(i)}(y(i)): i \in I\right\}+\frac{\varepsilon}{2} \leq M^{u}(y)
$$

so that the equality in assertion i) cannot be true for $y$.

Let $X$ be a Banach space. Given $u$ in $S_{X}$, we say that the norm of $X$ is strongly subdifferentiable at $u$ if

$$
M^{u}(x)=\lim _{\alpha \rightarrow 0^{+}} \frac{\|u+\alpha x\|-1}{\alpha} \text { uniformly for } x \text { in } B_{X}
$$

(equivalently, if $\lim _{\alpha \rightarrow 0} \varphi(X, u, \alpha)=0$ ). Given a nonempty set $I$, we denote by $B(I, X)$ the Banach space of all bounded functions from $I$ to $X$. A straightforward consequence of Theorem 1 is the following.

Corollary 2 (1]). Let $X$ be a Banach space, and let $u$ be in $S_{X}$. For every nonempty set $I$, denote by $\hat{u}$ the element of $B(I, X)$ defined by $\hat{u}(i)=u$ for all $i$ in $I$. Then the following assertions are equivalent:

i) For every nonempty set $I$ and every $f$ in $B(I, X)$, the equality

$$
V(B(I, X), \hat{u}, f)=\overline{\mathrm{co}}(\bigcup\{V(X, u, f(i)): i \in I\})
$$

holds.

ii) There exists an infinite set I such that

$$
V(B(I, X), \hat{u}, f)=\overline{\mathrm{co}}(\bigcup\{V(X, u, f(i)): i \in I\})
$$

for every $f$ in $B(I, X)$.

iii) $V(B(\mathbb{N}, X), \hat{u}, f)=\overline{\mathrm{co}}(\bigcup\{V(X, u, f(n)): n \in \mathbb{N}\})$ for all $f$ in $B(\mathbb{N}, X)$.

iv) The norm of $X$ is strongly subdifferentiable at $u$.

Given a Banach space $X$, and a subset $D$ of $S_{X}$, we say that the norm of $X$ is uniformly strongly subdifferentiable on $D$ if

$$
M^{u}(x)=\lim _{\alpha \rightarrow 0^{+}} \frac{\|u+\alpha x\|-1}{\alpha} \text { uniformly for }(u, x) \text { in } D \times B_{X} .
$$

Proposition 3. Let $E$ be a topological space without nonempty open finite subsets (for instance, every topological $T_{1}$-space without isolated points), $X$ a Banach space, and $g: E \rightarrow S_{X}$ a continuous function. Then the following assertions are equivalent: 
i) For every $f$ in $B(E, X)$, the equality

$$
V(B(E, X), g, f)=\overline{\mathrm{co}}(\bigcup\{V(X, g(t), f(t)): t \in E\})
$$

holds.

ii) The norm of $X$ is uniformly strongly subdifferentiable on the range of $g$.

Proof. ii) $\Rightarrow$ i) The uniform strong subdifferentiability of the norm of $X$ on the range of $g$ implies $\lim _{(t, \alpha) \rightarrow(\infty, 0)} \varphi(X, g(t), \alpha)=0$. Now, apply Theorem [1]

i) $\Rightarrow$ ii) Let $\varepsilon>0$. By assumption i) and Theorem [1 there exist $\delta>0$ and a co-finite subset $J$ of $E$ such that the inequality

$$
\frac{\|g(t)+\alpha x\|-1}{\alpha}-M^{g(t)}(x) \leq \frac{\varepsilon}{2}
$$

is true whenever $t$ is in $J, x$ is in $B_{X}$, and $0<\alpha<\delta$. Then, for $0<\alpha<\delta$, $0<\beta<\delta, t$ in $J$, and $x$ in $B_{X}$, we have

$$
\left|\frac{\|g(t)+\alpha x\|-1}{\alpha}-\frac{\|g(t)+\beta x\|-1}{\beta}\right| \leq \varepsilon
$$

Since $J$ is dense in $E$ and $g$ is continuous, the last inequality remains true for $0<\alpha<\delta, 0<\beta<\delta, t$ in $E$, and $x$ in $B_{X}$. By letting $\beta \rightarrow 0$, we obtain

$$
\frac{\|g(t)+\alpha x\|-1}{\alpha}-M^{g(t)}(x) \leq \varepsilon
$$

whenever $t$ is in $E, x$ is in $B_{X}$, and $0<\alpha<\delta$.

We note that Proposition 3 generalizes Corollary 2, Indeed, if $I$ is an infinite set, and if we endow $I$ with the trivial topology, then $I$ becomes a topological space with no nonempty open finite subsets, and the unique continuous functions on $I$ are the constant ones.

Let $X$ be a Banach space. Recall that, given $u$ in $S_{X}$, the norm of $X$ is said to be Fréchet differentiable at $u$ if there exists a continuous $\mathbb{R}$-linear mapping $\tau(u, \cdot): X \rightarrow \mathbb{R}$ such that

$$
\lim _{\|h\| \rightarrow 0} \frac{|\|u+h\|-1-\tau(u, h)|}{\|h\|}=0 .
$$

It is easy to see that the norm of $X$ is Fréchet differentiable at $u$ if and only if $X$ is smooth at $u$ (i.e., $D(X, u)$ reduces to a singleton) and the norm of $X$ is strongly subdifferentiable at $u$. If this is the case, then the unique element $\phi$ in $D(X, u)$ and the real-valued functional $\tau(u, \cdot)$ are mutually determined by means of the equality $\operatorname{Re} \circ \phi=\tau(u, \cdot)$. If the norm of $X$ is Fréchet differentiable at every point of $S_{X}$, and if we have

$$
\lim _{\|h\| \rightarrow 0} \frac{|\|u+h\|-1-\tau(u, h)|}{\|h\|}=0 \text { uniformly for } u \text { in } S_{X},
$$

then we say that $X$ is uniformly smooth. The notion of uniform smoothness of $X$ is apparently stronger than that of uniform strong subdifferentiability of the norm of $X$ on $S_{X}$, but, as proved in [4] Proposition 4.1], both notions actually are equivalent. Noticing also that the uniform strong subdifferentiability of the norm of $X$ on some subset $D$ of $S_{X}$ implies the uniform strong subdifferentiability of the norm of $X$ on the closure of $D$ (argue like in the proof of Proposition [3), we derive the next corollary. 
Corollary 4. Let $E$ be a topological space without nonempty open finite subsets, $X$ a Banach space, and $g$ a continuous function from $E$ onto a dense subset of $S_{X}$. Then the following assertions are equivalent:

i) For every $f$ in $B(E, X)$, the equality

$$
V(B(E, X), g, f)=\overline{\mathrm{co}}(\bigcup\{V(X, g(t), f(t)): t \in E\})
$$

holds.

ii) $X$ is uniformly smooth.

Taking in Corollary $4 E=S_{X}$ and $g$ equal to the inclusion mapping $S_{X} \hookrightarrow X$, we obtain the characterization of uniformly smooth Banach spaces announced in the introduction.

Theorem 5. For a Banach space $X$, the following assertions are equivalent:

i) For every bounded function $f: S_{X} \rightarrow X$, the equality $\overline{\operatorname{co}} W(f)=V(f)$ is true.

ii) $X$ is uniformly smooth.

To conclude the paper, let us show an elemental example of a Banach space $X$, together with a bounded function $f: S_{X} \rightarrow X$, such that the inclusion $\overline{c o} W(f) \subseteq$ $V(f)$ is strict.

Example 6. Take $X=\mathbb{R}^{2}$ with norm $\|(\lambda, \mu)\|:=\max \{|\lambda|,|\mu|\}$. For $x, y$ in $X$, put ]$x, y\left[:=\{\alpha x+(1-\alpha) y: 0<\alpha<1\}\right.$, and define a function $f: S_{X} \rightarrow X$ by

$$
f(u):= \begin{cases}(0,0) & \text { if } u \in\{(1,1),(1,-1),(-1,-1),(-1,1)\}, \\ (0,1) & \text { if } u \in](1,1),(1,-1)[\cup](-1,-1),(-1,1)[, \\ (1,0) & \text { if } u \in](1,-1),(-1,-1)[\cup](-1,1),(1,1)[.\end{cases}
$$

Then $f$ is bounded with $\|f\|=1$. Moreover, it is easily checked that the equality $\|\mathbf{1}+\alpha f\|=1+|\alpha|$ holds for every $\alpha$ in $\mathbb{R}$, and therefore $V(f)$ is equal to the closed real interval $[-1,1]$. However, we have $V(X, u, f(u))=\{0\}$ for every $u$ in $S_{X}$, and hence $W(f)=\{0\}$.

\section{ACKNOWLEDGEMENTS}

The author is most grateful to J. Becerra, R. Payá, and W. Werner for fruitful discussions about the matter in this note. He also thanks the referee for his interesting comments.

\section{REFERENCES}

[1] C. Aparicio, F. Ocaña, R. Paya, and A. Rodriguez, A nonsmooth extension of Fréchet differentiability of the norm with applications to numerical ranges, Glasgow Math. J. 28 (1986), 121-137. MR 87j:46026

[2] R. Deville, G. Godefroy, and V. Zizler, Smoothness and renormings in Banach spaces, Pitman Monographs and Surveys in Pure and Applied Math. 64, 1993. MR 94d:46012

[3] N. Dunford and J. T. Schwartz, Linear operators, Part I, Interscience Publishers, New York, 1958. MR 90g:47001a

[4] C. Franchetti and R. Paya, Banach spaces with strongly subdifferentiable norm, Bolletino U. M. I. 7-B (1993), 45-70. MR 94d:46015 
[5] L. A. Harris, The numerical range of holomorphic functions in Banach spaces, Amer. J. Math. 93 (1971), 1005-1019. MR 46:663

[6] G. Lumer, Semi-inner-product spaces, Trans. Amer. Math. Soc. 100 (1961), 29-43. MR 24A:2860

Departamento de Análisis Matemático, Universidad de Granada, 18071 Granada, SPAIN

E-mail address: apalacio@goliat.ugr.es 